\title{
FDTD Computation of Space/Time Integrated Electromagnetic Lagrangian: New Insights on Mutually Coupled Wide-band Antenna Design
}

\author{
Debdeep Sarkar and Yahia Antar
}

July 2021

\begin{abstract}
In this paper, we develop a formalism based on either spatially or temporally integrated electromagnetic (EM) Lagrangian, which provides new insights about the near-field reactive energy around generic antennas for arbitrary spatio-temporal excitation signals. Using electric and magnetic fields calculated via FDTD technique and interpolation routines, we compute and plot the normalized values of space/time integrated EM Lagrangian around antennas. While the time-integration of EM Lagrangian sheds light onto the spatial distribution of inductive/capacitive reactive energy, time-variation of spatially integrated EM Lagrangian can help in design of ultra-wideband (UWB) MIMO antennas with low mutual coupling. The EM Lagrangian approach can assist in design of energy harvesting and wireless power transfer systems, as well as for electromagnetic interference mitigation applications.
\end{abstract}

\section{Introduction}

The subject of understanding the radiation mechanism of antennas (from accelerated charge and/or time-varying current models), propagating energy flow and reactive (or "stored") energy dynamics around antennas, has intrigued researchers over many decades [1]-[6]. Consequently, the concepts of electromagnetic inertia, reactive time, localized waves and energy flow velocity are being developed to shed more light onto the deeper spatio-temporal dynamics of EM energy in the vicinity of radiating structures $[\underline{3},[\underline{5}$. Also, considerable emphasis has been imparted on the use of analytical techniques that can generate closed form expressions for fields, energy and associated quantities for well-known current distributions [1, 6]. Very recently, the concept of Poynting Localized energy was introduced and applied for antennas modelled in FDTD (finite-difference time-domain) framework, with the aim of evaluating space-time non-propagating energy around antennas $7 .-9$. It was highlighted in 9 that, although the traditional Q-factor approach can assist in quantifying the antenna impedance matching as well as determining the temporal evolution of total reactive

\footnotetext{
${ }^{1}$ Debdeep Sarkar (Corresponding Author) is with the Indian Institute of Science, Bangalore, 560012, Karnataka, India (debdeep@iisc.ac.in).

${ }^{2}$ Yahia Antar is with the Royal Military College, PO Box 17000, Station Forces Kingston, ON, K7K 7B4, Canada (antar-y@rmc.ca).
} 
energy in some specific cases, it is important to look beyond Q-factors to focus on the general spatio-temporal energy dynamics in particular.

While the localised energy concept was used for accurate prediction of gain-enhancement in strongly coupled Yagi-Uda type antenna systems [8, it did not provide the finer details like identifying the dominant/contributing fields (electric/magnetic) for energy localization. Furthermore, localized energy signatures are also used to perform mutual coupling path tracking at a preliminary level [9, a problem that is also addressed by several other research groups by means like Poynting streamlines, energy parcels and reaction theorem [10]-12. However, there lies ample scope for deploying additional constructs to further address the mutual coupling links between multiple antennas in MIMO arrays, or possible electromagnetic interference (EMI) mitigation [13]-14]. In 15. EM Lagrangian and it's intricate connection with the energy flow velocity (also explored in [2], [4]) is revisited. The possible use of EM Lagrangian and flow velocity in assessing the transitions from radiating (forward/backward propagating) to electrostatic/magnetostatic field regimes was strongly advocated in 15. However, the numerical examples under consideration in 15 mainly use analytica $\left.\right|^{1}$ space-time current (eg. Exponentially decaying) or field expressions, which may not be obtained in closed form for many realistic applied EM problems.

In this paper, we first compute the near-zone electric and magnetic fields from time-marching FDTD scheme, and then evaluate the aforementioned EM Lagrangian around antennas with arbitrary excitation. We further utilize quantities based on spatially or temporally integrated EM Lagrangian in order to: (i) obtain crucial understanding about the inductive/capacitive reactive energy distribution around antennas, (ii) identify regions of inter-antenna mutual coupling and near-field energy exchange, and (iii) faciltiate the design of coupled ultra-wideband (UWB) antenna systems with low mutual coupling. To the best of the authors' knowledge, the concepts of spatial or temporal integration of FDTD-computed EM Lagrangian, and its consequent application in analysis of two-port systems comprising of narrow-band thin-wire dipoles and UWB planar monopoles, are reported for the first time.

\section{Theory of Electromagnetic Lagrangian and Integration with FDTD}

In the context of general spatio-temporal electromagnetic fields, the Lagrangian $L=L(\mathbf{r}, t)$ density at any point having space-time varying electric field $\mathbf{E}=\mathbf{E}(\mathbf{r}, t)$ and magnetic field $\mathbf{H}=\mathbf{H}(\mathbf{r}, t)$ is formally defined as [15]:

$$
L=\frac{1}{2} \varepsilon \mathbf{E} \cdot \mathbf{E}-\frac{1}{2} \mu \mathbf{H} \cdot \mathbf{H},
$$

where $\varepsilon$ and $\mu$ respectively stand for the permittivity and permeability of the concerned medium. Using the Hamiltonian or total energy density $u=u(\mathbf{r}, t)$, we can get the normalized Lagrangian $l=l(\mathbf{r}, t)$ as follows:

$$
l=\frac{L}{u}, \text { where } u=\frac{1}{2} \varepsilon \mathbf{E} \cdot \mathbf{E}+\frac{1}{2} \mu \mathbf{H} \cdot \mathbf{H} .
$$

As discussed in [15], one can have a simple characterization of radiating and reactive fields using the following mapping of normalized Lagrangian $l$ : (a) $l \rightarrow 0$ : Pure, far-field EM wave, (b) $l \rightarrow+1$ :

\footnotetext{
${ }^{1}$ Note that, analytical solutions to general EM problems (eg. analysis and design of complex shaped printed/wire antennas) are not always feasible, and one needs to use computational EM tools like Finite Element Methods (FEM), Method of Moments (MoM), FDTD etc.
} 
Electrostatic field and (d) $l \rightarrow-1$ : Magnetostatic field. On the other hand, the energy flow velocity $\mathbf{v}_{e}=\mathbf{v}_{e}(\mathbf{r}, t)$ at that point is given by [4]:

$$
\mathbf{v}_{e}=\frac{\mathbf{E} \times \mathbf{H}}{\frac{1}{2} \varepsilon \mathbf{E} \cdot \mathbf{E}+\frac{1}{2} \mu \mathbf{H} \cdot \mathbf{H}},
$$

We can also represent the normalized energy flow velocity $v=v(\mathbf{r}, t)$ as,

$$
v=\frac{\left|\mathbf{v}_{e}\right|}{c}, \text { where } c=1 / \sqrt{\mu \varepsilon} .
$$

Note that, for far-zone propagating EM fields, one would have $v \rightarrow 1$, and otherwise for the reactive EM fields, $v$ would be reduced (i.e. slowing down of energy-flow). The importance of the Lagrangian-energy velocity space ("Great Electromagnetic Circle") regarding intuitive characterization of non-propagating/propagating EM fields, especially in the antenna near-zone, is ably highlighted in [15. However, in this letter, we would mainly focus on the EM Lagrangian ( $L$ and l) around antenna systems.

Note that, to calculate $l$ from respectively (2), one would require evaluation of $\mathbf{E}$ and $\mathbf{H}$ at same spatial location and time-instant; however in the staggered Yee grid, mathematical operations (i.e. cross-product, summation etc) $\mathbf{E}$ and $\mathbf{H}$ cannot be performed in a straightforward manner [19]. Therefore, one generally resorts to interpolation schemes [19], for evaluation of time-averaged Poynting flow [20] and Poynting localized energy [8]. Once the space-time values of $L=L(x, y, z, t)$ are obtained from a time-marching FDTD routine and suitable interpolation scheme, it is judicious to use temporal and spatial integration via operators $\mathcal{T}\{\star\}$ and $\mathcal{S}\{\star\}$, where:

$$
\begin{gathered}
\mathcal{T}\{L\}=\int_{0}^{T_{\mathrm{m}}} L(x, y, z, t) d t, \\
\mathcal{S}\{L\}=\int_{x_{\min }}^{x_{\max }} \int_{y_{\min }}^{y_{\max }} \int_{z_{\min }}^{z_{\max }} L(x, y, z, t) d x d y d z,
\end{gathered}
$$

Here $T_{\mathrm{m}}$ stands for the maximum FDTD computation time. Using the approach of [21, we define the quantities $\bar{l}$ and $\langle L\rangle$ as follows:

$$
\bar{l}=\frac{\mathcal{T}\{L\}}{\mathcal{T}\{u\}} \text { and }\langle L\rangle=\frac{\mathcal{S}\{L\}}{\max [\mathcal{S}\{L\}]}
$$

Note that, $\bar{l}$ in (7) is not equal to $\mathcal{T}\{l\}$ where $l=L / u$ (see 21 for more discussions). However, it can be shown that the inequalities $-1 \leq \bar{l} \leq 1$ and $-1 \leq\langle L\rangle \leq 1$ hold for any antenna system facing a finite duration temporal excitation (rigorous proof to be provided elsewhere). Also, the EM Lagrangian based quantities $\bar{l}$ and $\langle L\rangle$ can provide valuable insights regarding the space-time dynamics of antenna reactive energy (capacitive nature if $\langle L\rangle \rightarrow+1, \bar{l} \rightarrow+1$, and inductive nature if $\langle L\rangle \rightarrow-1, \bar{l} \rightarrow-1)$.

\section{Results of Numerical Computation on Coupled Antenna Systems (Narrow-band and UWB)}

\subsection{Example-I: System of Two Narrow-band Dipoles}

Fig. 1(a) shows the schematic diagram of a thin-wire center-fed dipole-based two-port configuration placed in a FDTD framework based on Cartesian co-ordinates. We use rectangular grid size of 
$\Delta x=\Delta y=\Delta z=\Delta=1 \mathrm{~mm}$ and total problem space as $70 \Delta \times 70 \Delta \times 70 \Delta$, bearing $10 \Delta$ padding of Berenger's PML (Perfectly matched layers) along each face. Following the CFL limit for stability [18, we choose $\Delta t=1.588$ ps. The thin-wire PEC dipoles are implemented by enforcing the $z$-component of electric field to be zero in the required locations.

We first estimate the S-parameters of two-dipole system of Fig. 1(a), by exciting the dipoles-1 and 2 using Gaussian pulse $p_{\mathrm{g}}(t)$ as:

$$
p_{\mathrm{g}}(t)=\exp \left[-\frac{\left(t-\tau_{1}\right)^{2}}{2 \sigma_{1}^{2}}\right],
$$

with $\tau_{1}=100 \Delta t, \sigma_{1}=4 \Delta t$. To feed the dipoles, "Delta-gap" mechanism is used [18, and when one dipole is excited by the temporal signal, the other one is kept terminated in matched load resistor in the Delta-gap region. Total simulation time $T_{m}=3000 \Delta t$ is chosen, so that the transient signals due to pulse excitation die out significantly and post-processing is not erroneous or misleading.

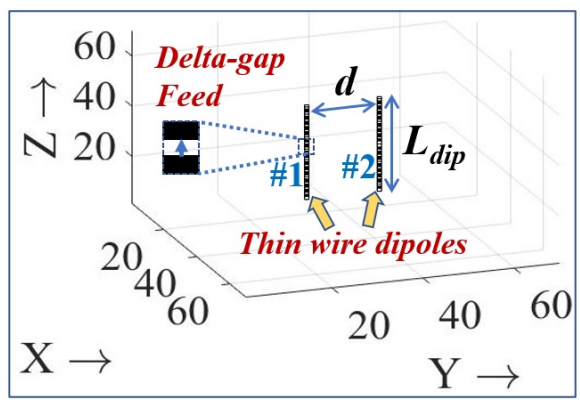

(a)

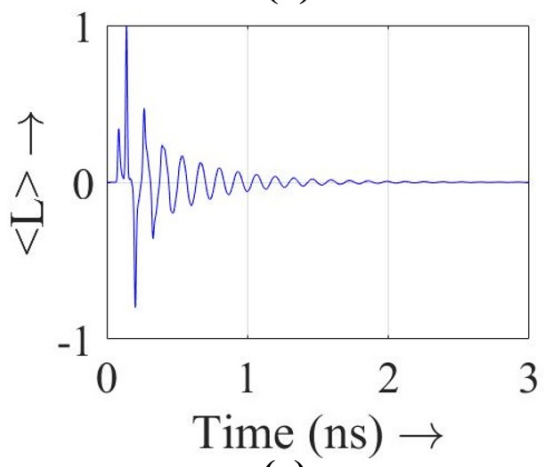

(c)

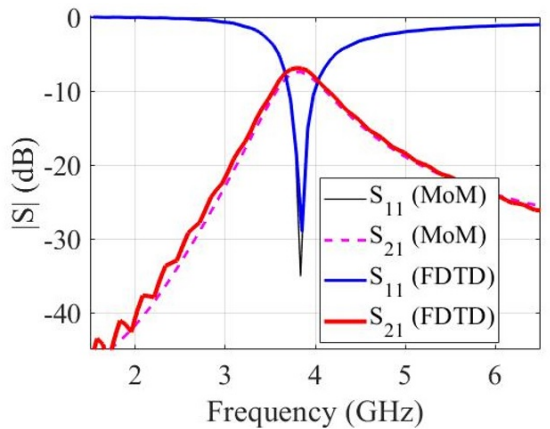

(b)

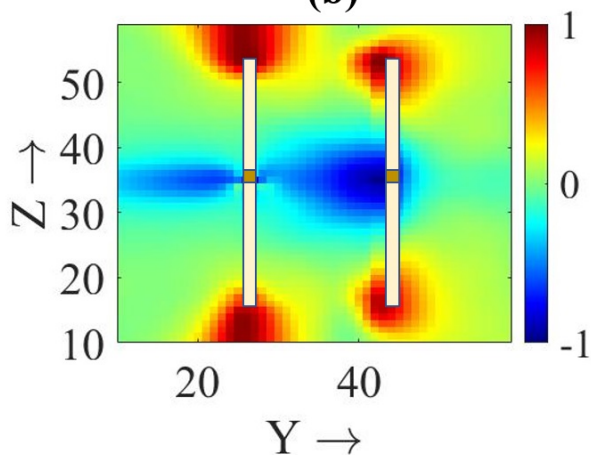

(d)

Figure 1: (a) Schematic diagram of the two dipole configuration, with $L_{d i p}=36 \mathrm{~mm}$ and $d=16$ mm (Delta feed-gap shown in zoomed view). (b) Simulated $S$-parameter magnitude computed using in-house FDTD and MATLAB Antenna toolbox. When dipole-1 is excited via Gaussian pulse keeping dipole-2 terminated by matched load: (c) Time evolution of $\langle L\rangle$. (d) Spatial patterns of $\bar{l}$ in the plane containing the dipoles. Note that, dipole- 1 in the left is excited.

Using the procedure described in [22], the S-parameters are calculated and bench-marked (see Fig. 1.(b)) with respect to MATLAB Antenna toolbox based on Method-of-Moments (MoM) [23]. 

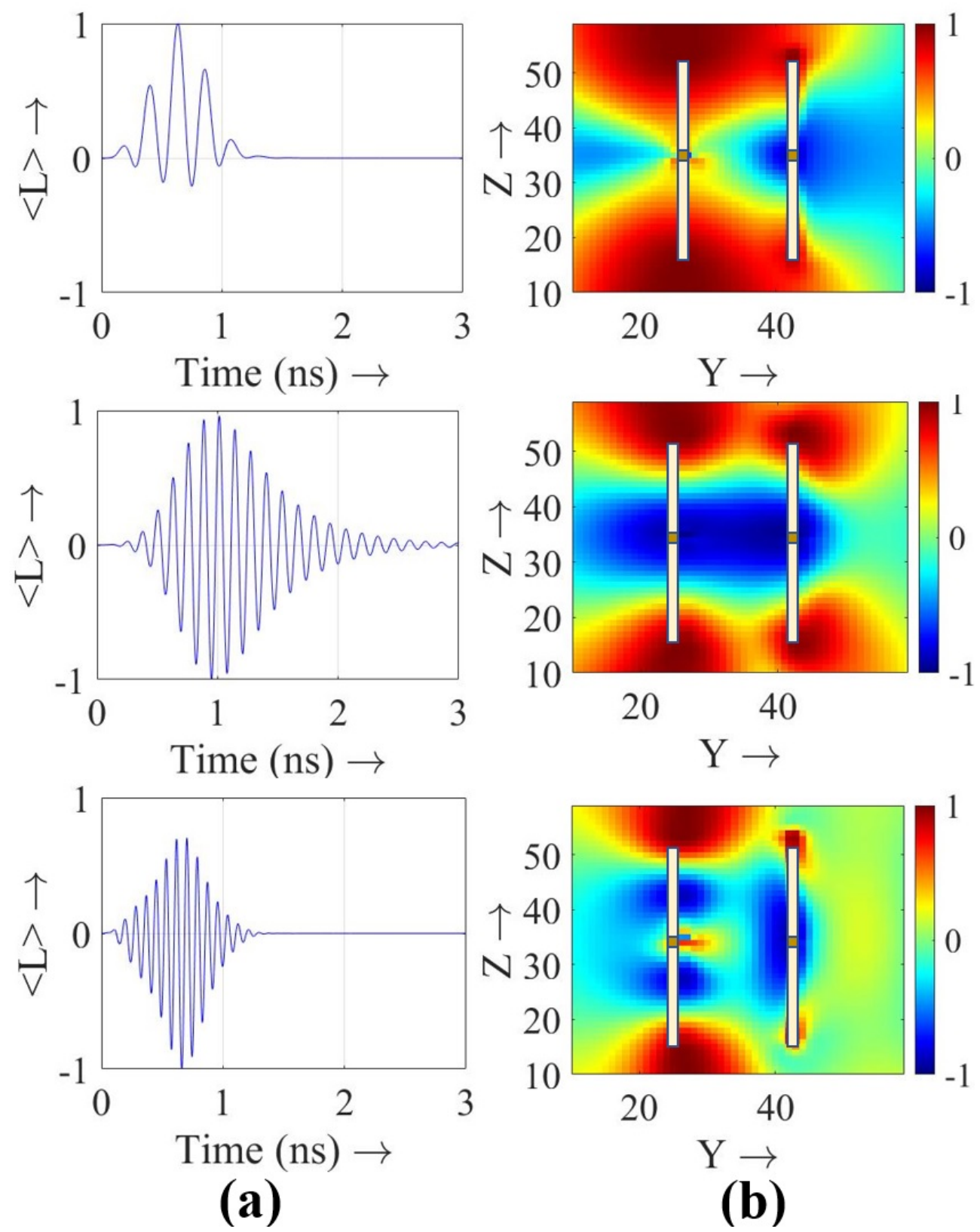

Figure 2: Simulated variation of: (a) $\langle L\rangle$ with time, (b) $\bar{l}$ along the $y z$-plane containing the dipoles for the system of Fig. 1(a). Dipole-1 is excited for modulated Gaussian pulse (see (9p) with $f_{0}$ values at $2 \mathrm{GHz}$ (top), $4 \mathrm{GHz}$ (middle) and $6 \mathrm{GHz}$ (bottom) in both Fig. 2(a) and Fig. 2(b). Also for clarity, images of the two dipoles are superimposed in spatial maps of $l$. Note that, dipole- 1 in the left is excited. 
Fig. 1(b) suggests that the two-dipole system shows narrow-band resonant characteristics with $\left|S_{11}\right|=-29.18 \mathrm{~dB}$ at $3.86 \mathrm{GHz}$ with $\left|S_{11}\right| \leq-10 \mathrm{~dB}$ impedance matching range $3.66-4.01 \mathrm{GHz}$. Also, high mutual coupling $\left(\left|S_{21}\right|=-6.86 \mathrm{~dB}\right)$ is observed as the separation between the two dipoles is only $0.206 \lambda$ ( $\lambda$ being the free-space wavelength at $3.86 \mathrm{GHz}$ ). Fig. 1(c) and Fig. 1(d) respectively demonstrate the time evolution of $\langle L\rangle$ along with spatial distribution of $\bar{l}$ in the plane containing the dipole. Although the Gaussian pulse bears wide-band frequency content, the two-dipole system has a narrow-band response (Fig. 1(b)). So the Gaussian pulse excites the fundamental resonant mode of the two-dipole system, and oscillations between capacitive and inductive reactive energy over the time can be observed from the temporal fluctuations of $\langle L\rangle$ (Fig. 1(c)).

Next, we consider modulated Gaussian pulse (typically considered in indoor channel-modelling problems [20]) given by $p_{\mathrm{mg}}(t)$ as the excitation signal:

$$
p_{\mathrm{mg}}(t)=\exp \left[-\frac{\left(t-\tau_{2}\right)^{2}}{2 \sigma_{2}^{2}}\right] \sin \left(2 \pi f_{0} t\right),
$$

where $\tau_{2}=600 \Delta t, \sigma_{2}=200 \Delta t$. Fig. 2(a) and Fig. 2(b) respectively show the time-evolution of $\langle L\rangle$ and spatial maps of $\bar{l}$ around the two-dipole system, for excitation frequencies $f_{0}=2,4$ and $6 \mathrm{GHz}$ respectively. When dipole- 1 is excited by $p_{\mathrm{mg}}(t)$ with $f_{0}=2.0 \mathrm{GHz}$, we have $0 \leq\langle L\rangle \leq 1$ most of the time, indicating that for this frequency, the reactive energy distribution around the antenna system is pre-dominantly capacitive (Fig. 2(a)), with very less spatial region having inductive nature (indicted by $\bar{l} \rightarrow-1$ ). This also corroborated from the $\bar{l} \rightarrow 1$ around the dipoles (especially near the arm tips see Fig. 2(b)). This is anticipated as we are operating in electrically small antenna regime $(k a=0.825<1)$, where $k=2 \pi f_{0} / c$ with $f_{0}=2.0 \mathrm{GHz}$, and $a=\sqrt{\left(L_{d i p} / 2\right)^{2}+(d / 2)^{2}}$ is radius of the smallest sphere (see Fig. 1(b)) circumscribing the two-dipole system.

When $f_{0}=4.0 \mathrm{GHz}$ is chosen, the value of $\langle L\rangle$ oscillates in equal manner above 0 -value within the range of $[-1,1]$ (Fig. 2(a)). This is due to the fact that $4 \mathrm{GHz}$ is close to the dipole impedance matching point (Fig. 1 (b)) and the resonant nature of the narrowband coupled dipole means that the system reactive energy goes back-and-forth between inductive and capacitive nature. While near the dipole-tips, one can observe $\bar{l} \rightarrow+1$ signifying high concentration of capacitive reactive energy, the region between the two dipole centers demonstrate strong complementary inductive energy concentration with $\bar{l} \rightarrow-1$. The spatial maps of $\bar{l}$ for $f_{0}=4 G H z$ in Fig. 2(b) further illustrate the strong mutual coupling and near-zone energy exchange links between the two side-byside dipoles. Finally as $f_{0}$ is increased to $6 \mathrm{GHz}$, the asymmetric temporal variation of $\langle L\rangle$ above the zero-level implies that fundamental dipole resonant mode is not excited at this frequency. Also, the reduction in the port-to-port mutual coupling is reflected from the spatial maps of regions having $\bar{l} \rightarrow 1$ and $\bar{l} \rightarrow-1$ around the individual dipoles, which no longer show a connected characteristics like the case with $f_{0}=4 \mathrm{GHz}$ (see Fig. 2(b)).

\subsection{Example-II: System of Two Wideband Monopoles}

It is important to note that the proposed concept of spatially or temporally integrated EM lagrangian based quantities $\langle L\rangle$ and $\bar{l}$ can be applied for any general antenna system modelled in an FDTD regime. Fig. 3(a) shows a planar monopole based two-port system, analyzed within $1-12$ $\mathrm{GHz}$ in the in-house FDTD regime. The fundamental antenna operating frequency (around 2.35 $\mathrm{GHz}$ in this case) is governed by length $L_{m}$ of the planar monopoles, and the wide-band S-parameter response also indicates the presence of third order harmonic around $7 \mathrm{GHz}$ (see Fig. 3(b)). The monopole width ( $W_{m}$ for the structure in Fig. 3(a)), can be tailored to realize wide impedance 


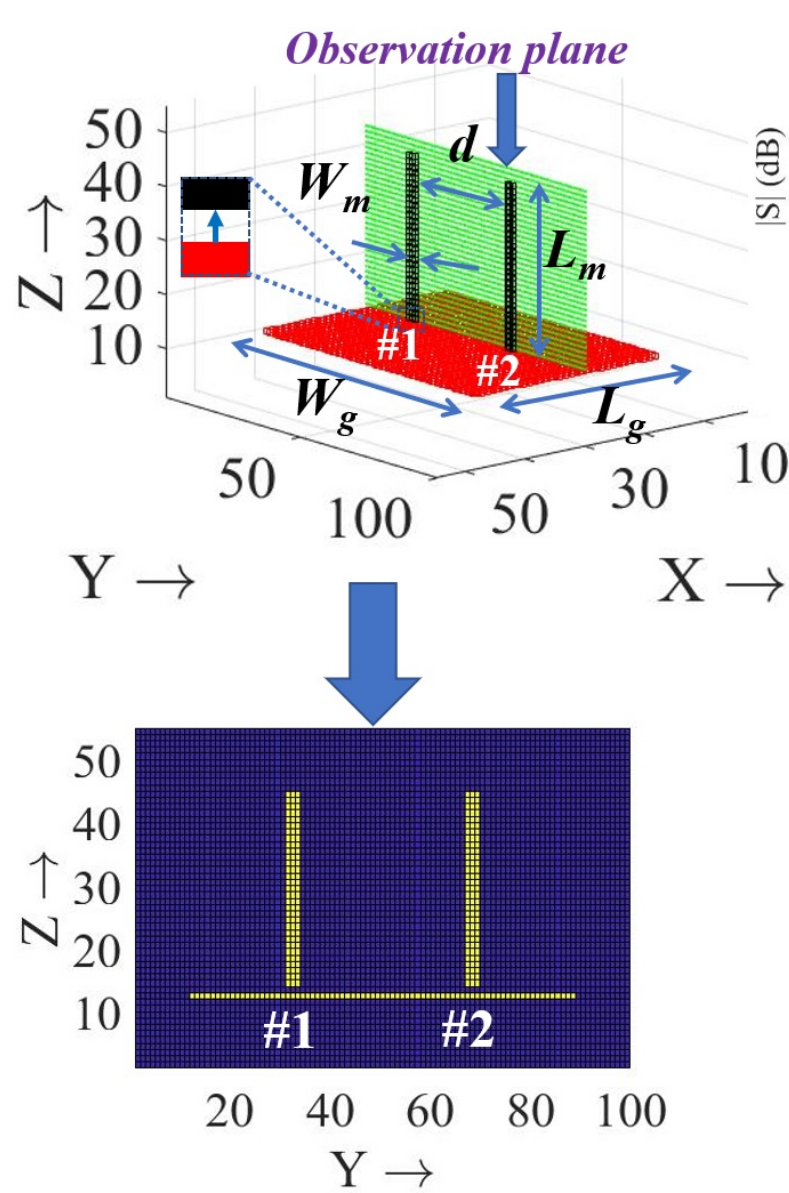

(a)

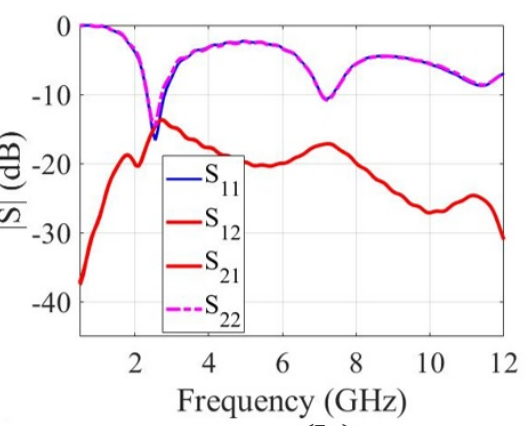

(b)

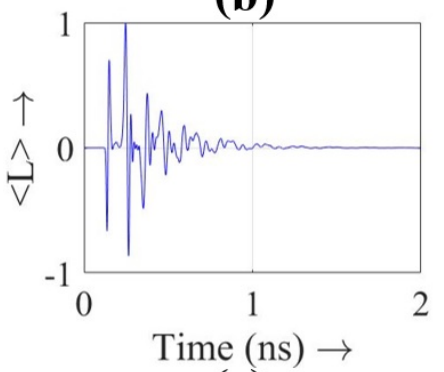

(c)

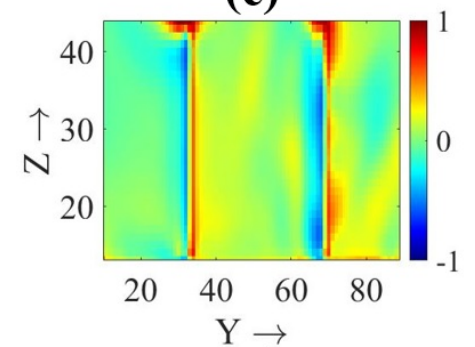

(d)

Figure 3: (a) Schematic diagram of a two-port planar monopole antenna system with dimensions (in mm.): $W_{m}=3, d=34, W_{g}=76, L_{g}=30$. The cross-sectional view of the plane containing the two monopoles is also shown below for better clarity. The observation plane for $\bar{l}$ spatial patterns is chosen $2 \mathrm{~mm}$ away (along $x$-direction) from the two monopoles, (b) Variation of S-parameter magnitude with frequency for the two-port system. Exciting monopole-1 is excited by Gaussian pulse and keeping monopole-2 terminated in matched load: (c) Temporal variation of $\langle L\rangle$, (d) spatial pattern of $\bar{l}$ along the observation plane. 

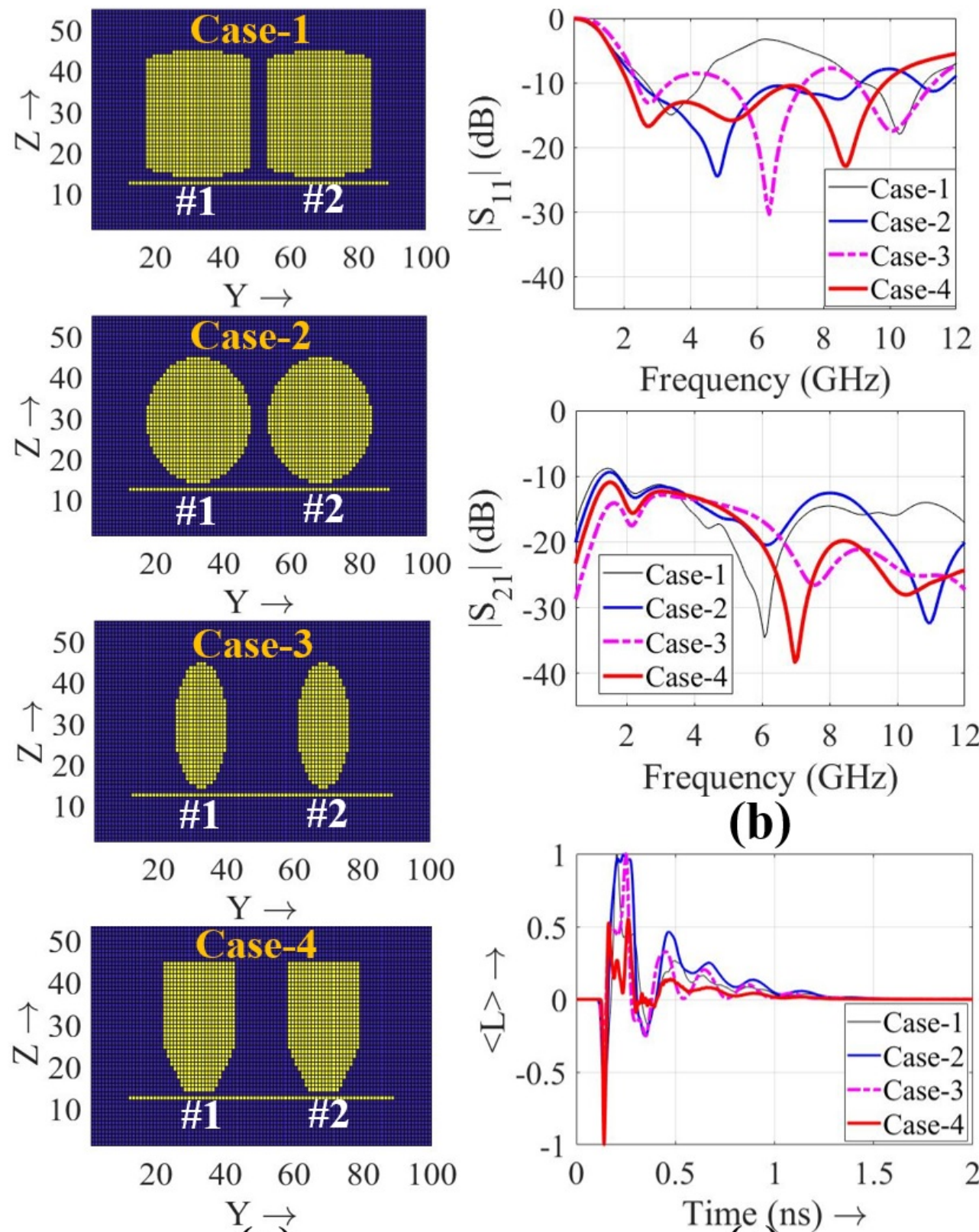

(a)

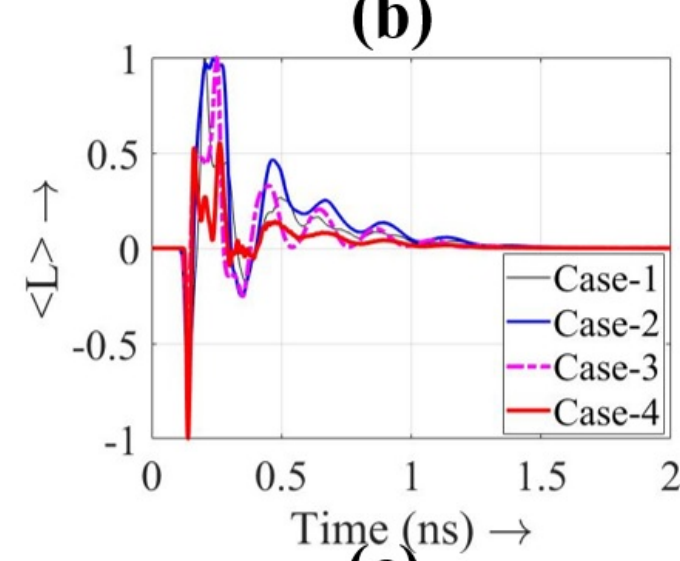

(c)

Figure 4: (a) Few possible two-port configurations based on planar monopoles, realized by modifying the reference geometry in Fig. 3(a). (b) Comparative frequency variations of $\left|S_{11}\right|$ (top) and $\left|S_{21}\right|$ (top) for the structures (Case-I to Case-IV) in Fig. 4(a). (c) Comparison between temporal variations of $\langle L\rangle$ for the two-port configurations (Case-I to Case-IV), when antenna-1 is excited by a Gaussian pulse and antenna-2 is terminated by matched load. 
matching response. Fig. 3(c) shows that he temporal variation $\langle L\rangle$ for Gaussian pulse excitation $\left(\tau_{1}=100 \Delta t, \sigma_{1}=10 \Delta t\right)$ of monopole-1 has significant amount of oscillatory ripples. Intuitively, it can be said that narrow-band resonant systems will show more rapid fluctuations between inductive and capacitive reactive energy, when excited by a short-duration temporal pulse.

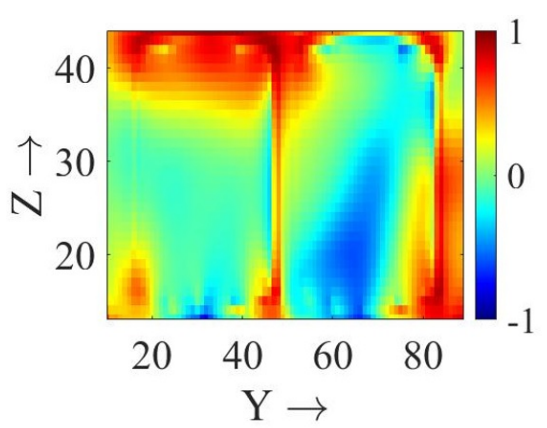

(a)

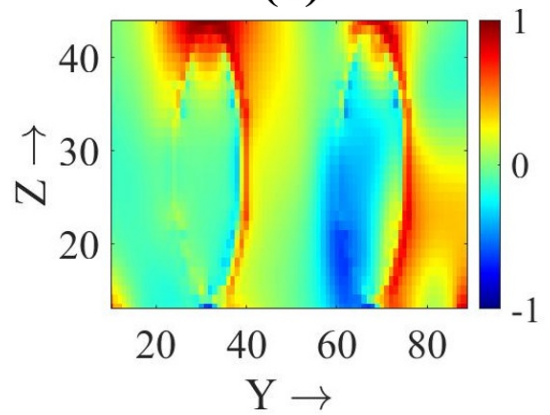

(c)

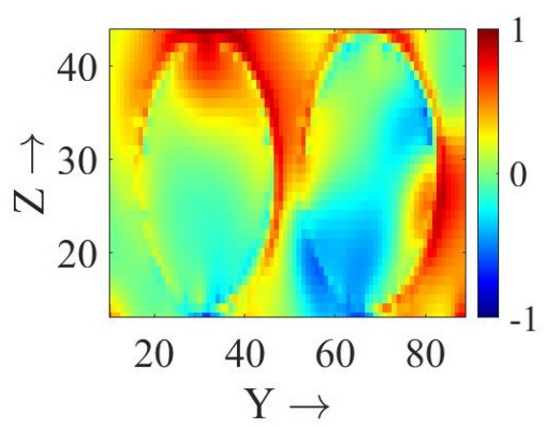

(b)

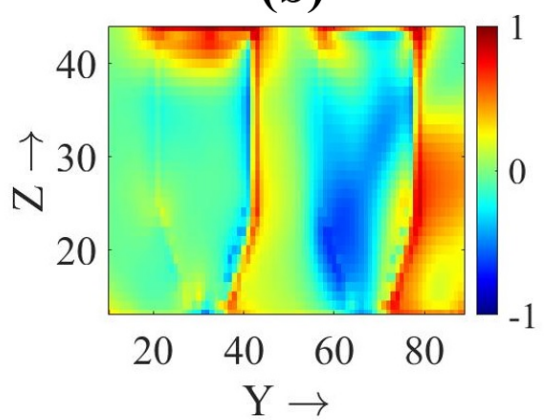

(d)

Figure 5: Spatial patterns of $\bar{l}$ along the observation-pulse for the configurations of Fig. 4(a): (a) Case-I, (b) Case-II, (c) Case-III and (d) Case-IV. Antenna-1 is excited by Gaussian pulse with Antenna-2 terminated in matched load.

Next, we present four planar antennas by modifying the basic configuration of Fig. 3(a) that can be used for two-port UWB system design targeting the popular FCC-specified 3.1-10.6 GHz band (Fig. 4(a)). As anticipated, the planar "fat" monopoles show enhanced multi-band/UWB impedance matching behaviour (Fig. 4(b)); moreover, the frequency variation of mutual coupling $\left(\left|S_{21}\right|\right)$ depends on the relative position and edge-to-edge separation of the UWB radiating structures. The structure in Case- 4 of Fig. 4 (a) has the best performance (see Fig. 4(b)) in terms of the impedance matching $(<-10 \mathrm{~dB}$ in the desired band of $3.1-10.6 \mathrm{GHz})$ as well as port-to-port coupling (especially $<-20 \mathrm{~dB}$ in the frequency range $>6 \mathrm{GHz}$ ). Now, the corresponding timevariation of $\langle L\rangle$ (choosing Gaussian pulse excitation) reveals that the design of Case- 4 has the least temporal fluctuations in $\langle L\rangle$ (Fig. 4(c)).

Also, Fig. 5 indicates that, for the UWB system in Case-4 (optimum mutual coupling and matching performance among the four cases), the near-zone capacitive reactive energy concentration (indicated by $\bar{l} \rightarrow 1$ ) is minimal (Fig. 5(d)). Therefore, $\langle L\rangle$ and $\bar{l}$ around coupled wide-band antennas bear a fundamental connection with both impedance matching and mutual coupling (note 
that, both are related to the reactive energy distribution around closely spaced antennas).

\section{Conclusion}

We utilise FDTD computed $\mathbf{E}$ and $\mathbf{H}$ fields along with suitable interpolation routines to compute EM Lagrangian based quantities, $\langle L\rangle$ and $\bar{l}$, around arbitrary antenna systems for any temporal excitation. Note that, simple plots of near-zone electric and magnetic fields around antennas do not give idea about purely reactive energy distribution, as near-zone fields have both propagating and non-propagating components. Though the traditional Q-factor approach gives some idea about time-variation of the reactive energy, the spatial distribution aspect of non-propagating energy around antennas is not emphasized in the circuit-approach. While, the Poynting Localized energy distribution can plot the non-propagating energy around antennas [8], the EM Lagrangian approach using $\bar{l}$ provides a unique way to spatially distinguish regions containing inductive and capacitive reactive energy. Furthermore, minimization of both $\langle L\rangle$ and $\bar{l}$ around coupled antenna topologies can help in the design of UWB MIMO systems with high inter-port isolation, based on optimization of pixelated antenna geometries. The EM Lagrangian approach can be used in EMI analysis, as well as energy harvesting and wireless power transfer applications.

\section{References}

[1] H. G. Schantz, "Electromagnetic energy around Hertzian dipoles," IEEE Antennas and Propagation Magazine, vol. 43, no. 2, pp. 50-62, April 2001.

[2] G. Kaiser, "Electromagnetic Inertia, Reactive Energy and Energy Flow Velocity," Journal of Physics A: Mathematical and Theoretical, vol. 44, no. 34, 345206, Aug. 2011.

[3] G. Kaiser, "Conservation of Reactive EM energy in Reactive time," Proceedings of 2015 IEEE International Symposium on Antennas and Propagation and USNC/URSI National Radio Science Meeting (APSURSI), pp. 1704-1705, 2015.

[4] P. Saari, O. Rebane, and I. Besieris, "Energy-flow velocities of non-diffracting localized waves," Physical Review A, vol. 100, 013849, pp. 1-10, 2019.

[5] P. Saari and I. Besieris, "Reactive energy in non-diffracting localized waves," Physical Review A, vol. 101, 023812, pp. 1-9, 2020.

[6] M. S. Mirmoosa, G. A. Ptitcyn, R. Fleury, S. A. Tretyakov, "Instantaneous radiation from time-varying electric and magnetic dipoles," Physical Review A, vol. 102, 2020.

[7] S. Mikki, D. Sarkar and Y. Antar, "On Localized Antenna Energy in Electromagnetic Radiation," Progress in Electromagnetics Research M, vol. 79, pp. 1-10, March 2019.

[8] D. Sarkar, S. Mikki and Y. Antar, "Poynting Localized Energy: Method and Applications to Gain Enhancement in Coupled Antenna Systems," IEEE Transactions on Antennas and Propagation, vol. 68, no. 5, pp. 3978-3988, May 2020.

[9] D. Sarkar, S. Mikki and Y. Antar, "Representation of Electromagnetic Energy around Antennas: Current Research and Future Directions," IEEE Antennas and Propagation Magazine, 2021. (In Press) 
[10] J. Diao, L. Liu and K. F. Warnick, "An Intuitive Way to Understand Mutual Coupling Effects in Antenna Arrays Using the Poynting Streamline Method," IEEE Transactions on Antennas and Propagation, vol. 67, no. 2, pp. 884-891, 2019.

[11] H. Li, V. V. Khilkevich and D. Pommerenke, "Identification and Visualization of Coupling Paths-Part I: Energy Parcel and Its Trajectory," IEEE Transactions on Electromagnetic Compatibility, vol. 56, no. 3, pp. 622-629, 1 June 2014.

[12] J. Malmstrom, H. Holter and B. L. G. Jonsson, "On Mutual Coupling and Coupling Paths Between Antennas Using the Reaction Theorem," IEEE Transactions on Electromagnetic Compatibility, vol. 60, no. 6, pp. 2037-2040, Dec. 2018.

[13] Y. Zhong, W. Song, C. Kim and C. Hwang, "Coupling Path Visualization and Its Application in Preventing Electromagnetic Interference," IEEE Transactions on Electromagnetic Compatibility, vol. 62, no. 4, pp. 1485-1492, Aug. 2020.

[14] A. Talebzadeh et al., "Coupling Path Visualization and EMI Mitigation for Flyover QSFP Connectors," IEEE Transactions on Electromagnetic Compatibility, vol. 62, no. 4, pp. 10371044, Aug. 2020.

[15] H. G. Schantz, "Energy velocity and reactive fields," Philosophical Transactions A (Royal Society), vol. 376, 20170453, 2018.

[16] K. S. Yee, "Numerical solution of initial boundary value problems involving Maxwell's equations in isotropic media," IEEE Transactions on Antennas and Propagation, vol. AP-14, no. 3, pp. 302-307, May 1966.

[17] A. Taflove, Computational Electrodynamics: The Finite Difference Time Domain Method, London, U.K.: Artech House, 1995.

[18] D. M. Sullivan, Electromagnetic Simulation Using the FDTD Method, New York, NY, USA:IEEE Press, 2000.

[19] R. Kastner and O. Asaf, "Energy conservation and Poynting's theorem in the staggered FDTD grid," Proc. URSI Gen. Assembly, pp. 1-4.

[20] D. Sarkar, S. Mikki and Y. Antar, "Engineering the Eigenspace Structure of Massive MIMO Links Through Frequency-Selective Surfaces," IEEE Antennas and Wireless Propagation Letters, vol. 18, no. 12, pp. 2701-2705, December 2019.

[21] C. A. Valagiannopoulos and A. Alu, "The Role of Reactive Energy in the Radiation by a Dipole Antenna," IEEE Transactions on Antennas and Propagation, vol. 63, no. 8, pp. 37363741, Aug. 2015.

[22] D. Sarkar and K. V. Srivastava, "Application of Cross-correlation Green's Function along with FDTD for Fast Computation of Envelope Correlation Coefficient over Wideband for MIMO Antennas," IEEE Transactions on Antennas and Propagation, vol. 65, no. 2, pp. 730-740, February 2017.

[23] Antenna Toolbox by MATLAB. https://in.mathworks.com/products/antenna.html 